Pathologe 2012 · 33:118-123

DOI 10.1007/s00292-011-1549-5

Online publiziert: 18. Februar 2012

(c) Springer-Verlag 2011
C. Rosamilia - G. Feichter · A. Tzankov - E.C. Obermann

Institut für Pathologie, Universitätsspital Basel, Schweiz

\title{
Diagnose und Graduierung zervikaler intraepithelialer Neoplasien
}

\section{Hintergrund \\ Histopathologie}

Grundlage für das Management von Patientinnen mit (prä-)neoplastischen Veränderungen der Portio ist die zytologische bzw. histopathologische Diagnostik. Die Einteilung der Dysplasien der Portio erfolgt nach der Systematik der World Health Organization (WHO) in zervikale intraepitheliale Neoplasien Grad 1, 2 und 3. Diese Systematik basiert in erster Linie auf einer subjektiven Abschätzung der Ausdehnung der dysplastischen Veränderungen im Verhältnis zur gesamten Breite des Epithels [33]. Diese dreigliedrige Einteilung berücksichtigt neuere Erkenntnisse nicht, dass es sich bei den intraepithelialen Läsionen um histologisch erkennbare, durch das humane Papillomavirus (HPV) induzierte Veränderungen handelt, welche zwei unterschiedliche Prozesse umfassen, nämlich eine benigne Alteration sowie eine präneoplastische Läsion [35]. Bei einer CIN2 handelt es sich wahrscheinlich um eine heterogene Kategorie, die sich einerseits z. T. aus Fällen zusammensetzt, welche sich wie CIN1 mit Tendenz zur spontanen Regression verhalten. Andererseits handelt es sich aber um Läsionen, welche eher einer CIN3 nahestehen [7]. Die histopathologische Beurteilung von Läsionen der Portio unterliegt einer seit Langem bekannten Interobservervariabilität $[18,24]$.

Die korrekte Graduierung der CIN ist wichtig, da diese die Grundlage für weitere Therapieentscheidungen liefert. Nach den S2k-Leitlinien der Deutschen Gesellschaft für Gynäkologie und Geburtshilfe erfolgt, sofern auch Positivität für einen HPV vom High-risk-Typ besteht, ein konservatives Management mit regelmäßigen kolposkopisch-zytologischen Kontrollen für CIN1 über bis zu 24 Monate und für CIN2 bis zu 12 Monate. Bei CIN3 soll nach diesen Leitlinien eine Konisation durchgeführt werden [10]. Reaktive Veränderungen müssen von einer CIN1 abgegrenzt werden. Tendenziell erfolgt bei dieser Fragestellung eher eine Überinterpretation [29]. Seltener sind Überinterpretationen schwerer reaktiver Veränderungen nichtdysplastischen Epithels als CIN2/3 [6].

\section{Einsatz von Biomarkern}

Verschieden Studien befassten sich mit Biomarkern, welche die Diagnostik der CIN erleichtern könnten. Voraussetzung für die Anwendbarkeit in der Praxis ist jedoch, dass diese Marker ohne übermäBigen technischen Aufwand in der Routinediagnostik eingesetzt werden können. In dieser Studie analysierten wir an einer großen Serie von Portiobiopsien den HPV-assoziierten Marker p16 sowie die Proliferationsmarker Ki-67 und Mcm2.

Bei p16 handelt es sich um einen cyclinabhängigen Kinase(CDK)-Inhibitor der INK4-Familie, der als Tumorsuppressorprotein agiert. Eine erhöhte Expression von p16 lässt sich in (Prä-)Neoplasien der Portio nachweisen. Wahrscheinlich beruht dies auf einer funktionellen Inaktivierung von $\mathrm{pRB}$ durch das E7-Protein von HPV, was wiederum $\mathrm{zu}$ einer vermehrten Expression von p16 führt [2]. Eine Korrelation zwischen dem Grad einer CIN und der Expression von p16 war in früheren Untersuchungen gezeigt worden $[8,9,13,19,25,31]$.

$\mathrm{Ki}-67$ ist ein in der Histopathologie breit angewandter Proliferationsmarker. Dieses Protein wird in allen Phasen des Zellzyklus mit Ausnahme der G0- und frühen G1-Phase exprimiert [14] und wurde als hilfreich zur Abgrenzung reaktiver Veränderungen gegenüber einer CIN1 bzw. Graduierung der CIN beschrieben $[4,8,13,20,26]$.

Der Mcm-Komplex („minichromosome maintenance complex") ist aus den Mcm-Proteinen, den Schlüsselproteinen des präreplikativen Komplexes, aufgebaut und dient der Initiierung des Zellzyklus. Im Gegensatz zu Ki-67 werden Mcm-Proteine auch in der frühen G1-Phase exprimiert [17]. Insbesondere die Untergruppen $\mathrm{Mcm} 2$ und Mcm5 des Proteinkomplexes wurden bereits sehr früh als diagnostisch hilfreiche und prognostisch relevante Marker bei dysplastischen Epithelveränderungen propagiert $[12,34]$.

Andere Biomarker, welche ebenfalls bei der Zellteilung und/oder Dysregulation des Zellzyklus eine Rolle spielen, wurden bereits auf ihre Wertigkeit bei der Diagnostik der CIN untersucht, so z. B. Cyclin E, p21, RB (Retinoblastomprotein) und PCNA (,proliferating cell nuclear antigen"). Eine ganze Reihe weiterer Biomarker, wie z. B. CEA („carcinoembrionic antigen"), MN-Antigen [4], p63, bcl-2, [27], Gewebetransglutaminase [2], Survivin und MYBL2 [23] sowie die Zytokeratine 13 und 14 als Differenzierungsmarker [21], wurden zwar in einzelnen Publikationen untersucht, konnten sich jedoch bisher in der Routinediagnostik nicht etablieren. 


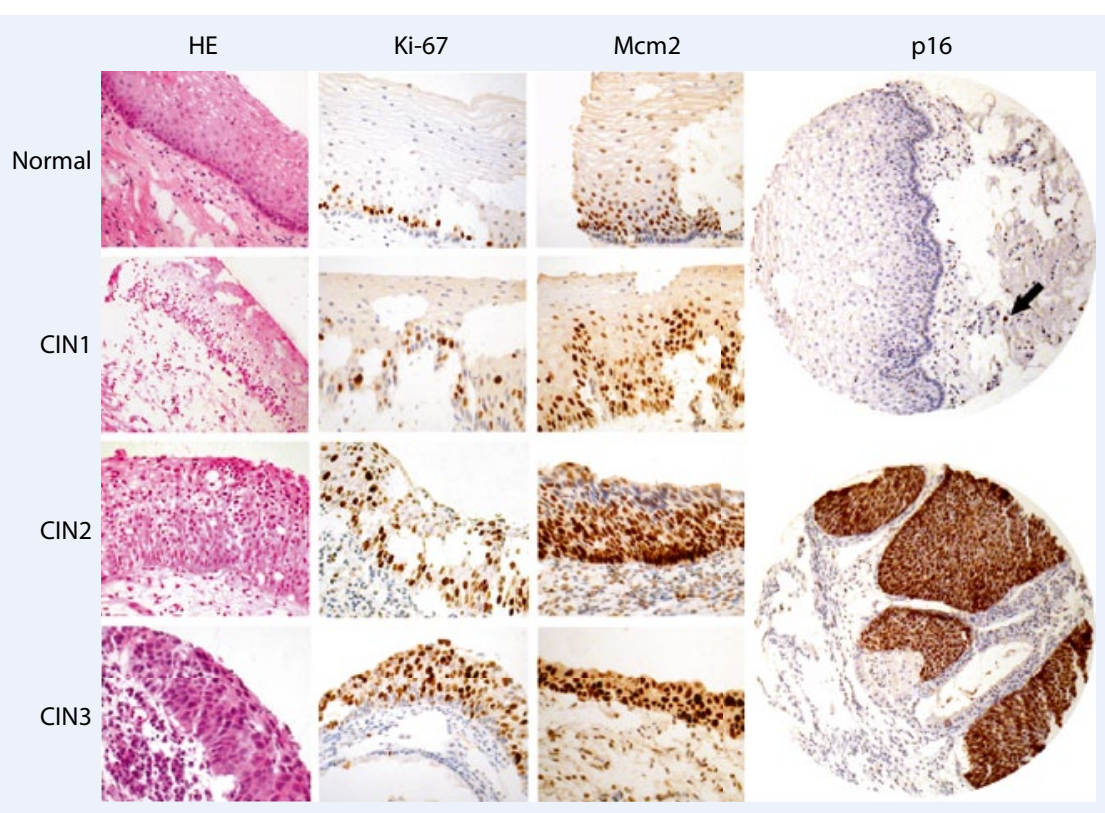

Abb. 1 D Dysplasiefreies Epithel und zervikale intraepitheliale Neoplasien (CIN) Grad 1 bis 3 in der HEFärbung (1. Zeile) sowie nach den immunhistochemischen Färbungen auf Ki-67 und Mcm2 (2. und 3. Zeile). Rechts oben: Stanzzylinder mit nichtdysplastischem p16-negativem Epithel. Pfeil: p16-positiver Lymphozyt als positive interne Kontrolle. Rechts unten: Stanzzylinder aus einer CIN3 mit diffuser kräftiger Positivität für p16

Ein anderer Ansatz ist der Nachweis von Biomarkern, die direkt den Status einer HPV-Infektion zeigen. Immunhistochemisch ist z. B. der Nachweis des L1Kapsids möglich, dessen Vorliegen eine produktive Virusinfektion beweist. Die Wertigkeit dieses Markers für die Graduierung der CIN ist jedoch umstritten [13, 25].

Der Nachweis einer onkogenen HPVInfektion durch die Detektion der viralen Proteine E6 und E7 bzw. deren Messenger-RNA, Untersuchungen zur Viruslast sowie die HPV-Typisierung könnten theoretisch in der Diagnostik der CIN hilfreich sein und darüber hinaus auch Informationen über das Progressionsrisiko geben. Die Anwendung in der Routinediagnostik an formalinfixierten, paraffineingebetteten Gewebeproben ist zum jetzigen Zeitpunkt jedoch nur eingeschränkt möglich $[5,9]$.

\section{Fragestellung}

Ziel dieser Studie war es, einen Marker bzw. ein Set von Markern zu identifizieren, deren/dessen Anwendung in der täglichen Routinediagnostik gut möglich ist und welche(r) bei der Diagnostik und evaluiert (E.O, C.R.).

\section{Immunhistochemie}

Die immunhistochemischen Analysen wurden unter Verwendung folgender Antikörper durchgeführt:

- Ki-67 (clone MIB-1, DAKO, Glostrup, Dänemark, Verdünnung 1:50),

- Mcm2 (CRCT2.1 Acris Antibodies, Herford, Deutschland, Verdünnung 1:100),

- p16 (clone E6H4, DAKO, Glostrup,

Dänemark, vorverdünnt).

Die gefärbten Schnitte wurden von C.R. und E.O. beurteilt. Abweichende Ergebnisse wurden am Diskussionsmikroskop diskutiert, bis eine Einigung erreicht wurde. Bei Ki-67 und Mcm2 wurde in Anlehnung an Duggan et al. [11] der prozentuale Anteil aller Zellen mit nukleärer Expression bezogen auf alle Epithelzellen im untersuchten Areal in 5\%-Schritten geschätzt.

Für die Auswertung von p16 wurden die Kriterien von Galgano et al. [13] und Klaes et al. [18] herangezogen. Somit wurde Positivität für p16 dann angenommen, wenn eine starke diffuse Färbung zu erkennen war. Als starke Färbung galt nur eine eindeutig erkennbare zytoplasmatische und ggf. nukleäre Reaktion. Als diffuses Muster galt eine kontinuierliche, meist ab der Basalmembran beginnende Färbereaktion, welche sich über mehr als ein Drittel des Epithels erstreckte (• Abb. 1). In Übereinstimmung mit Klaes et al. [18] wurde eine fokale Positivität für p16 als negativ beurteilt.

\section{Statistische Auswertung} chen 1985 und 2000 am Institut für Pathologie der Universität Basel untersucht worden waren. Von den Paraffinblöcken wurde je ein HE-gefärbter (Hämatoxylin und Eosin) Schnitt angefertigt, in denen repräsentative Tumorareale selektiert wurden. Hieraus wurden zwei TMAs hergestellt [32]. Alle Proben auf den TMAs wurden mittels eines HE-Schnitts erneut

Für diese Studie wurden nur Fälle ausgewählt, in welchen die Diagnosen normales Epithel, CIN1, CIN2, CIN3 sowohl an den ursprünglichen Gewebeschnitten als auch an den HE-gefärbten Schnitten der TMAs übereinstimmten.
In die statistische Auswertung wurden nur Fälle einbezogen, bei welchen sowohl im HE-Schnitt des TMA als auch am ursprünglichen Schnittpräparat die gleiche Diagnose gestellt worden war. Unterschiede in der prozentualen Expression von Ki-67 bzw. Mcm 2 bei den unterschiedlichen Kategorien (nichtdysplastisches Epithel, CIN1, CIN2, CIN3) wurden mit dem nichtparametrischen WilcoxonRangsummentest evaluiert. Für die Auswertung der p16-Expression wurde der Fischer-Exakt-Test angewandt, wobei jeweils SAS (V9.1, The Sas Institute, Cary, N.Y, USA) zur Anwendung kam. 
Die Bestimmung der optimalen Markerkombination erfolgte mit der CARTAnalyse („classification and regression tree analysis“, DTREG Predictive Modeling Software, www.dtreg.com). Der mit dieser Methode erstellte computergenerierte Algorithmus benutzt einen binären rekursiven Prozess, durch den die Daten in bestmögliche Kombinationen von Variablen zur optimalen Klassifizierung gebracht werden. Zur Messung des Klassifikationsfehlers als Funktion der Entscheidungsbaumgröße wurden 10-fache Crossvalidierungsuntersuchungen durchgeführt. Proben, zu denen keine Expressionsdaten vorlagen, wurden von der Analyse ausgeschlossen.

\section{Ergebnisse}

Bei 297 Gewebeproben auf den TMAs war sowohl am ursprünglichen Gewebeschnitt als auch am TMA eine identische Diagnose gestellt worden. In 27 Fällen handelte es sich um dysplasiefreies Plattenepithel, in 31 Fällen um eine CIN1, in 83 Fällen um eine CIN2 und in 156 Fällen um eine CIN3.

Die durchschnittlichen Anzahl der proliferierenden Zellen betrug

- im nichtdysplastischen Epithel ge-

messen an Ki-67 13\% (Range: 0-30\%)

und an Mcm2 62\% (Range: 10-90\%),

- bei CIN1 gemessen an Ki-67 22\%

(Range: 5-70\%) und an Mcm2 65\%

(30-95\%),

- bei CIN2 gemessen an Ki-67

$37 \%$ (o-90\%) und an $\mathrm{Mcm} 282 \%$

(40-95\%),

- bei CIN3 gemessen an Ki-67

51\% (o-90\%) und an Mcm2 86\%

(50-95\%).

Sowohl für Ki-67 als auch für Mcm2 zeigte sich eine Zunahme der durchschnittlichen Werte der proliferierenden epithelialen Zellen von den Proben mit nichtdysplastischem Epithel über CIN1, CIN2 bis zur CIN3. In dem paarweisen Vergleich lag sowohl für Ki-67 als auch für Mcm2 ein statistisch signifikanter Unterschied von $\mathrm{p}<0,001$ vor (• Tab. 1).

Die Unterscheidung zwischen einer CIN1 und einer CIN2 war mittels Ki-67 möglich. Bei einer Proliferationsrate von $<25 \%$ gemessen mittels Ki-67 handelte

Pathologe 2012 · 33:118-123 DOI 10.1007/s00292-011-1549-5

(C) Springer-Verlag 2011

\section{Rosamilia · G. Feichter · A. Tzankov · E.C. Obermann}

\section{Diagnose und Graduierung zervikaler intraepithelialer Neoplasien}

\section{Zusammenfassung}

Diagnose und Graduierung von zervikalen intraepithelialen Neoplasien (CIN) gehören zu den häufigen Fragestellungen in der histopathologischen Diagnostik. Trotzdem kann die Unterscheidung zwischen reaktiven Veränderungen und CIN1 bzw. die Graduierung einer CIN Schwierigkeiten bereiten.

In dieser Studie wurde untersucht, ob die Bestimmung der Proliferationsmarker Ki-67 und $\mathrm{Mcm} 2$ sowie von $\mathrm{p} 16$ zur Beantwortung dieser Fragestellungen beitragen. Untersucht wurden die immunhistochemischen Expressionsprofile dieser Marker an 297 Proben aus dysplasiefreiem Portioepithel, CIN1, CIN2 und CIN3 mittels Gewebemikroarrays.

Die mittels Ki-67 bzw. Mcm2 ermittelte Proliferationsrate zeigte eine Zunahme von dysplasiefreiem Epithel über CIN1, CIN2 zu CIN3 ( $p<0.001$ bei beiden Markern). Mittels Ki-67 ließ sich am besten zwischen dysplasiefreiem Epithel und CIN1 unterscheiden. Zur Abgrenzung von CIN1 zu CIN2 bot sich eine
Kombination von Ki-67 und p16 an. Bei einer Ki-67-Expression von $<25 \%$ handelte es sich um eine CIN1 mit einer Sensitivität von 91,7\% und einer Spezifität von 54,3\%. Die zusätzliche Untersuchung der Expression von p16 konnte einen weiteren Teil der Fälle mit einer Ki-67-Expression von $<25 \%$ stratifizieren. Die Anzahl p16-positiver Fälle betrug bei normalen Epithelien 0\%, bei CIN1 7\%, bei CIN2 46\% und bei CIN3 86\%. Somit war mittels p 16 eine Abgrenzung der CIN2 zur CIN3 nur zum Teil möglich.

Die histopathologische Evaluation am HESchnitt bleibt Grundlage der Beurteilung von zervikalen intraepithelialen Neoplasien, jedoch kann die Analyse von Ki-67 und p16 zur Diagnose und Graduierung beitragen.

Schlüsselwörter

Zervikale intraepitheliale Neoplasie ·

Proliferationsmarker $\cdot \mathrm{p} 16 \cdot \mathrm{Mcm} 2 \cdot \mathrm{Ki}-67$

\section{Diagnosis and grading of cervical intraepithelial neoplasias}

\section{Abstract}

Diagnosing and grading of cervical intraepithelial neoplasias (CIN) are part of the routine practice of pathologists. However, discriminating between reactive changes and CIN1 and determining the different degrees of CIN may be challenging. Aim of this study was the evaluation of the proliferation markers Ki67 and Mcm2 as well as p16 for their potential to aid in the assessment of CIN.

297 samples of normal epithelium, CIN1, CIN2, and CIN3 were assessed for expression of the above mentioned markers using tissue microarrays.

There was an increase in the expression of Ki67 and Mcm2 from normal epithelium, CIN1, CIN2 to CIN3 ( $p<0.001$ for both markers). Ki-67 was the most useful marker in differentiating between normal epithelium and CIN1. The number of p16-positive cases was $7 \%$ in CIN1, $46 \%$ in CIN2 and $86 \%$ in CIN3. There were no p16-positive cases in the group with normal epithelium. In order to grade CIN1 vs. CIN2 a combination of Ki67 and p16 was helpful. Cases with a proliferation rate of $<25 \%$ assessed with $\mathrm{Ki}-67$ were most likely CIN1 (sensitivity $91.7 \%$, specificity: $54.3 \%$, positive predictive value: $73.3 \%$, negative predictive value $82.6 \%$ ). P16 was the most helpful marker in distinguishing between $\mathrm{CIN} 2$ and $\mathrm{CIN} 3$ as p16 negative cases were more likely to belong into the CIN2 category.

In summary, the histopathological assessment of cervical biopsies is based on H\&Estained slides. However, Ki- 67 and $\mathrm{p} 16$ can be helpful in diagnosing and grading cervical intraepithelial neoplasia.

\section{Keywords}

Cervical intraepithelial neoplasia .

Proliferation markers - Cyclin-dependent kinase inhibitor p16 $\cdot$ Mcm 2 protein · Ki-67 es sich um eine CIN1 mit einer Sensitivität von $91,7 \%$ und einer Spezifität von $54,3 \%$. Der positive prädiktive Wert von Ki-67 betrug für diese Fragestellung $73,3 \%$, der negative prädiktive Wert lag bei $82,6 \%$. Die zusätzliche Untersuchung der Expression von p16 konnte einen weiteren Teil der Fälle mit einer Ki-67-Expression von $<25 \%$ stratifizieren: Bei p16positiven Fällen handelte es sich nur um CIN2. Die Sensitivität einer fehlenden Expression von p16 in der Gruppe der Fälle 
Tab. 1 Expression von Ki-67 und Mcm2 bei dysplasiefreiem Epithel und zervikalen intraepithelialen Neoplasien (CIN) Grad 1 bis 3

\begin{tabular}{|lllllc}
\hline & Normal (n=27) & CIN1 (n=31) & CIN2 (n=83) & CIN3 (n=156) & p-Wert \\
\hline Ki-67 & $13 \%(0-30 \%)$ & $22 \%(5-70 \%)$ & $37 \%(0-90 \%)$ & $51 \%(0-90 \%)$ & $<0,001$ \\
\hline Mcm2 & $62 \%(10-90 \%)$ & $65 \%(30-95 \%)$ & $82 \%(40-95 \%)$ & $86 \%(50-95 \%)$ & $<0,001$ \\
\hline $\begin{array}{l}\text { Angabe des durchschnittlichen Prozentwerts der Ki-67- bzw. Mcm2-exprimierenden Zellen an allen epithelialen Zellen im un- } \\
\text { tersuchten Areal. Angaben zur Streuung (Range) in Klammern. }\end{array}$
\end{tabular}

Tab. 2 Expression von p16 bei dysplasiefreiem Epithel und zervikalen intraepithelialen Neoplasien (CIN) Grad 1 bis 3

\begin{tabular}{|llllll} 
& Normal (n=27) & CIN1 (n=30) & CIN2 (n=80) & CIN3 (n=150) & p-Wert \\
\hline Negativ & $100 \%(27)$ & $93 \%(28)$ & $54 \%(43)$ & $14 \%(21)$ & $<0,001$ \\
\cline { 1 - 3 } Positiv & $0 \%(0)$ & $7 \%(2)$ & $46 \%(37)$ & $86 \%(129)$ & \\
\cline { 1 - 2 } Angabe der Häufigkeit als Prozent in der jeweiligen Kategorie; absolute Fallzahlen in Klammern. &
\end{tabular}

Angabe der Häufigkeit als Prozent in der jeweiligen Kategorie; absolute Fallzahlen in Klammern.

mit einer Ki-67-Expression von $<25 \%$ betrug für die Diagnostik einer CIN1 57,6\%, die Spezifität lag hierfür bei $100 \%$.

Bei der Markierung mit p16 war bei den Fällen mit nichtdysplastischen Epithelien keine der 27 Proben positiv (0\%). Bei CIN1 zeigten 2 von 30 (7\%), bei CIN2 37 von 80 (46\%) und bei CIN3 129 von 150 (86\%) Fällen eine Positivität für p16. Weiterhin zeigte sich eine statistisch signifikante Zunahme der Anzahl p16-positiver Fälle mit Zunahme des Dysplasiegrads (p<0,001; - Tab. 2, • Abb. 1).

\section{Diskussion}

Die korrekte Diagnose und Graduierung zervikaler intraepithelialer Neoplasien dient als Grundlage des klinischen Managements der betroffenen Patientinnen. Einerseits besteht die Möglichkeit, Veränderungen zu niedrig zu klassifizieren, was dazu führen kann, dass die Patientin keine adäquate Therapie erhält. Eine Einordnung einer reaktiven Veränderung als CIN1 führt dagegen zu unnötigen Nachkontrollen und stellt möglicherweise eine Belastung für die Patienten dar. Die Diagnose einer CIN2 oder CIN3 kann sogar Eingriffe nach sich ziehen, welche die Fertilität beeinflussen können. Die histopathologische Diagnostik der CIN ist durch eine hohe Interobservervariabilität gekennzeichnet [18, 24]. Zahlreiche Arbeiten befassen sich daher mit der Frage, ob zusätzliche Parameter die konventionelle Diagnostik am HE-Schnitt unterstützen könnten.

Da eine Infektion mit HPV Voraussetzung für die Entstehung eines Platten- epithelkarzinoms der Portio und seiner Vorläuferläsionen ist, scheint es sinnvoll, Parameter zu untersuchen, welche unmittelbar mit einem HPV-Infekt in $\mathrm{Zu}$ sammenhang stehen. Zu diesen Parametern zählen z. B. die Messung der mRNATranskripte von E7 [9] oder die Untersuchungen zu HPV selbst, d. h. die Bestimmung der Viruslast, die Virustypisierung und der Nachweis einer Integration der viralen DNA [5]. Nachteil dieser Methoden ist der technische Aufwand, welcher die Anwendung in der Routinediagnostik limitiert. Eine weitere Möglichkeit für die Diagnostik der CIN ist die Analyse von Biomarkern, welche die Zellproliferation und/oder die Dysregulation des Zellzyklus erfassen, wie z. B. Ki-67, Mcm-Proteine, Cyclin E, PCNA und p16 [4].

\section{Nutzen von Biomarkern}

Ziel dieser Studie war es, Biomarker auf ihren Nutzen bei der Diagnostik und Graduierung der CIN in der täglichen Routinediagnostik zu untersuchen. Ausgewählt wurden die Marker Ki-67 und Mcm2, da sie die Proliferation bzw. das proliferative Potenzial des Epithels erfassen können. p16 wurde in die Untersuchung aufgenommen, da dessen Expression in unmittelbarem Zusammenhang mit einer HPV-Infektion steht. Ein wichtiges Auswahlkriterium der Marker war deren einfache Anwendbarkeit in der täglichen Diagnostik.

\section{Ki-67}

Insbesondere Ki-67 ist ein etablierter Proliferationsmarker, welcher routinemäßig in der histopathologischen Diagnostik für unterschiedliche Fragestellungen eingesetzt wird. Mcm2 wird schon seit Langem als Proliferationsmarker propagiert [30], konnte Ki-67 aber nie verdrängen.

Verschiedene Auswertungsmodalitäten wurden für diese Marker beschrieben, wie z. B. der Nachweis von Ki-67exprimierenden Zellclustern [20, 26] und/oder bildanalyseunterstützte Techniken [4]. Für diese Arbeit wurde in Anlehnung an Duggan et al. [11] der prozentuale Anteil markierter Zellen an allen Epithelien des untersuchten Areals bestimmt, da es sich hierbei um eine in der Praxis gut durchführbare Methode handelt. Hier zeigte sich, dass Ki-67 für die Abgrenzung des nichtdysplastischen Epithels (durchschnittliche Proliferationsrate: 13\%) von CIN1 (durchschnittliche Proliferationsrate: $22 \%$ ) nützlich sein kann. Dabei zeigen sich jedoch Überlappungen zwischen beiden Kategorien, so dass im Einzelfall eine sichere Unterscheidung schwierig bleibt (• Tab. 1).

Auch andere Autoren konnten eine diagnostische Wertigkeit von Ki-67 für die Diagnose einer CIN nachweisen. Bei der Auswertung sind allerdings v. a. intraepitheliale Entzündungszellen und tangentielle Anschnitte der Basalzellschicht zu beachten [13, 20, 26]. Insbesondere bei entzündlich-reaktiven Veränderungen des Epithels kann die Proliferationsrate erhöht sein, so dass die Abgrenzung dieser Läsionen zu einer CIN1 allein auf Grund der Proliferationsrate nur eingeschränkt möglich ist [1].

\section{Mcm2}

Unsere Untersuchung zeigte auch eine $\mathrm{Zu}$ nahme der Expression von $\mathrm{Mcm} 2$ von normalem Epithel über CIN1 und CIN2 zu CIN3. Ein Vorteil von $\mathrm{Mcm} 2$ gegenüber Ki-67 bestand jedoch nicht. Möglicherweise ist ProExC (Tripath Imaging Inc., Burlington, NC), welches einen Antikörpercocktail gegen $\mathrm{Mcm} 2$ - und Topoisomerase-II-alpha-Proteine enthält, einer alleinigen Untersuchung von $\mathrm{Mcm} 2$ bei der Diagnostik zervikaler Läsionen überlegen [28].

In der Literatur wird zur Unterscheidung von nichtdysplastischen Epithelveränderungen, insbesondere der unreifen Plattenepithelmetaplasie und der schweren Atrophie, zu einer CIN neben der 


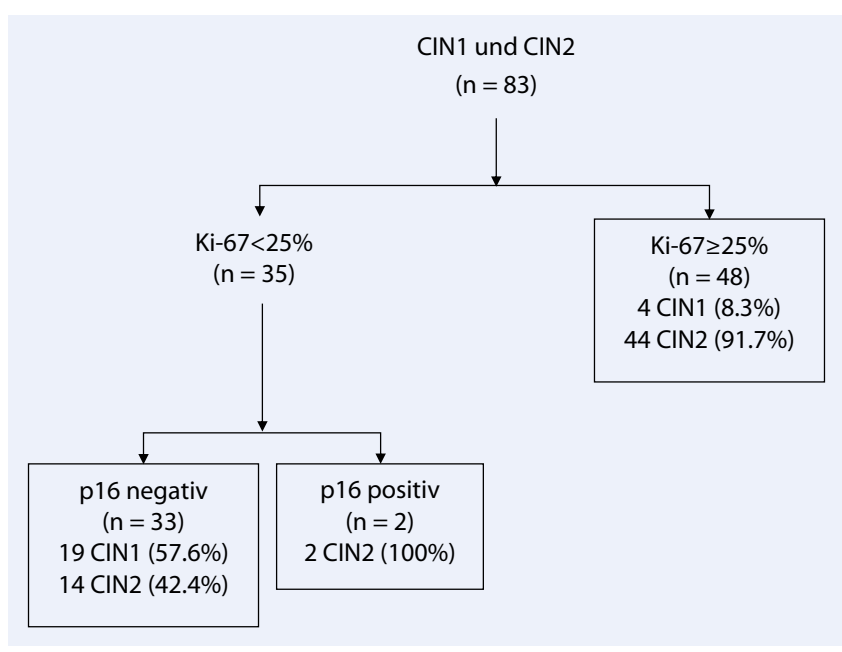

Abb. $2<$ Algorithmus zur Expression von Ki-67 und $\mathrm{p} 16$ bei zervikalen intraepithelialen Neoplasien (CIN) Grad 1 und 2

sorgfältigen morphologischen Diagnostik meist eine kombinierte Analyse von Ki-67 und p16, ggf. zusammen mit einer HPV-Testung, empfohlen [16].

\section{p16}

Die Wertigkeit von p16 für die Diagnostik zervikaler Läsionen ließ sich bereits in zahlreichen Studien zeigen $[9,13,31]$. Im Unterschied zu unserer Untersuchung wurden insbesondere bei einer CIN1 von anderen Autoren mehr Fälle mit Positivität für p16 beobachtet. Die Angaben in der Literatur reichen von $25 \%$ [15] bis zu $88 \%$ [18]. Auch für CIN2/CIN3 sind oft höhere Anteile an positiven Fällen als in unserer Studie beobachtet worden $[9,31]$.

Ursächlich hierfür können das Patientinnenkollektiv, der verwendete Antikörper und v. a. die unterschiedlichen Auswertungskriterien sein. In dieser Studie war nur eine diffuse, starke, eindeutig erkennbare Reaktion als positiv gewertet worden, da dieses Kriterium sowohl der Literatur entsprach [18] als auch für die Routinediagnostik geeignet schien. Auch wenn die Auswertungskriterien für die untersuchten immunhistochemischen Marker in dieser Studie im Einklang mit der Literatur standen, kann die fehlende Standardisierung der Analytik zwischen verschiedenen Laboratorien zu der unterschiedlichen Rate an p16-positiven Fällen beigetragen haben. Hierbei spielen sicher neben den möglichen Variablen im Labor auch präanalytische Faktoren (z. B. Art und Dauer der Gewebeprobenfixation) eine Rolle [22].

\section{Einsatz in der Graduierung}

Für die Graduierung der CIN1 im Unterschied zu einer CIN2 kann nach unserer Untersuchung in Ergänzung zur Morphologie eine kombinierte Untersuchung von Ki-67 und p16 hilfreich sein.

Hierbei bietet sich ein Algorithmus mit einer Evaluation von Ki-67 und p16 an, da mehr als 90\% aller CIN2 eine Ki-67-Expression von mindestens $25 \%$ aufweisen (Sensitivität: 91,7\%). Falls die Proliferationsrate $<25 \%$ ist, lassen sich weitere Fälle auf Grund ihrer Positivität für p16 einer CIN2 zuordnen (• Abb. 2).

Eine Abgrenzung zwischen CIN2 und CIN3 ist anhand der hier untersuchten Marker am besten mittels p16 möglich, da ein Großteil der Fälle (86\%) von CIN3 eine Expression von p16 zeigt, so dass eine Negativität für p16 bei dieser Fragestellung für eine CIN2 spricht. Umgekehrt kann bei Positivität für p16 jedoch nicht auf eine CIN3 geschlossen werden, da auch fast die Hälfte der CIN2 (46\%) p16 exprimiert. Die breite Streuung der mittels Ki-67 bzw. Mcm2 gemessenen Proliferationsrate und der p16Expression innerhalb der einzelnen Grade der CIN zeigt, dass bei der CIN nicht deutlich voneinander abgegrenzte Kategorien vorliegen. Vielmehr handelt es sich um einen dynamischen Prozess, der sowohl zur Regression als auch Progression fähig ist. Insbesondere die Gruppe der CIN2 ist wahrscheinlich heterogen zum einen aus Fällen zusammengesetzt, die in ihrem weiteren Verlauf der CIN1 zuzuordnen sind, sowie zum anderen aus Fällen, die sich wie CIN3 verhalten [7].
Zusammenfassend bleibt die morphologische Beurteilung am HE-Schnitt Grundlage für die Diagnostik und Graduierung der CIN. In einem Teil der Fälle kann die Untersuchung weiterer Marker, wie z. B. Ki-67 oder p16, hilfreich sein. In Zukunft werden wahrscheinlich Biomarker in den Vordergrund rücken, welche nicht nur die Diagnostik unterstützen, sondern auch prognostische Informationen über den zu erwartenden Verlauf der zervikalen intraepithelialen Neoplasien bieten.

\section{Fazit für die Praxis}

- Die korrekte Diagnose und Graduierung sind von klinischer Bedeutung für das Management von Patientinnen mit zervikalen intraepithelialen Neoplasien (CIN), jedoch können sie in der Routinediagnostik eine Herausforderung darstellen.

- Als Unterstützung zur histopathologischen Beurteilung kann sich der Proliferationsmarker Ki-67 für die Unterscheidung von nichtdysplastischem Epithel im Gegensatz zu einer CIN1 als hilfreich erweisen.

- Zur Abgrenzung einer CIN1 gegenüber einer höhergradigen Läsion dient eine Analyse von Ki-67 und, bei einer Proliferationsrate $<25 \%$, eine nachfolgende Untersuchung von p16. Bei Fällen mit niedriger Proliferationsrate und Negativität von p16 dürfte es sich um eine CIN1 handeln.

- Die Unterscheidung von CIN2 und CIN3 kann durch Untersuchung von p16 erleichtert werden.

- Insgesamt bleibt die Morphologie am HE-Schnitt Grundlage der Diagnostik und Graduierung der $\mathrm{CIN}$, allerdings können Zusatzuntersuchungen mittels Ki-67 und p16 in einem Teil der Fälle nützlich sein. 


\section{Korrespondenzadresse}

\section{PD Dr. E.C. Obermann}

Institut für Pathologie, Universitätsspital Basel

Schönbeinstr. 40, 4031 Basel

Schweiz

EObermann@uhbs.ch

Anmerkung

Die vorgestellten Ergebnisse sind Anteile der Dissertationsschrift von C.R.

Danksagung. Ganz besonderer Dank gilt Fr. PD Dr. I. Zlobec, Institut für Pathologie der Universität Bern für die statistische Auswertung und die hilfreichen Diskussionen. Für technische Unterstützung bedanken sich die Autoren bei Fr. T. Nguyen.

Interessenkonflikt. Der korrespondierende Autor gibt an, dass kein Interessenkonflikt besteht.

\section{Literatur}

1. Agoff SN, Lin P, Morihara J et al (2003) p16(INK4a) expression correlates with degree of cervical neoplasia: a comparison with Ki-67 expression and detection of high-risk HPV types. Mod Pathol 16:665673

2. Arends MJ, Buckley CH, Wells M (1998) Aetiology, pathogenesis, and pathology of cervical neoplasia. J Clin Pathol 51:96-103

3. Baak JP, Kruse AJ, Robboy SJ et al (2006) Dynamic behavioural interpretation of cervical intraepithelial neoplasia with molecular biomarkers. J Clin Pathol 59:1017-1028

4. Baldwin P, Laskey R, Coleman N (2003) Translational approaches to improving cervical screening. Nat Rev Cancer 3:217-226

5. Boulet GA, Horvath CA, Berghmans S, Bogers J (2008) Human papillomavirus in cervical cancer screening: important role as biomarker. Cancer Epidemiol Biomarkers Prev 17:810-817

6. Castle PE, Cox JT, Jeronimo J et al (2008) An analysis of high-risk human papillomavirus DNA-negative cervical precancers in the ASCUS-LSIL Triage Study (ALTS). Obstet Gynecol 111:847-856

7. Castle PE, Stoler MH, Solomon D, Schiffman M (2007) The relationship of community biopsy-diagnosed cervical intraepithelial neoplasia grade 2 to the quality control pathology-reviewed diagnoses: an ALTS report. Am J Clin Pathol 127:805-815

8. Conesa-Zamora, Domenech-Peris A, Orantes-Casado FJ et al (2009) Effect of human papillomavirus on cell cycle-related proteins p16, Ki-67, Cyclin D1, p53, and ProEx C in precursor lesions of cervical carcinoma: a tissue microarray study. Am J Clin Pathol 132:378-390

9. Cuschieri K, Wentzensen N (2008) Human papillomavirus mRNA and p16 detection as biomarkers for the improved diagnosis of cervical neoplasia. Cancer Epidemiol Biomarkers Prev 17:2536-2545

10. Deutsche Gesellschaft für Gynäkologie und Geburtshilfe, Arbeitsgemeinschaft Infektiologie und Infektimmunologie in Gynäkologie und Geburtshilfe, Berufsverband der Frauenärzte et al (2008) Prävention, Diagnostik und Therapie der HPV-Infektion und präinvasiver Läsionen des weiblichen Genitale: AWMF 015/027 (S2k) www.dggg.de/fileadmin/public docs/Leitlinien/g_01_04 04_praevention_diagnostik_therapie_hpvinfektion.pdf
11. Duggan MA, Akbari M, Magliocco AM (2006) Atypical immature cervical metaplasia: immunoprofiling and longitudinal outcome. Hum Pathol 37:1473-1481

12. Freeman A, Morris LS, Mills AD et al (1999) Minichromosome maintenance proteins as biological markers of dysplasia and malignancy. Clin Cancer Res 5:2121-2132

13. Galgano MT, Castle PE, Atkins KA et al (2010) Using biomarkers as objective standards in the diagnosis of cervical biopsies. Am J Surg Pathol 34:10771087

14. Gerdes J, Lemke H, Baisch H et al (1984) Cell cycle analysis of a cell proliferation-associated human nuclear antigen defined by the monoclonal antibody Ki-67. J Immunol 133:1710-1715

15. Ishikawa M, Fujii T, Saito M et al (2006) Overexpression of p16 INK4a as an indicator for human papillomavirus oncogenic activity in cervical squamous neoplasia. Int J Gynecol Cancer 16:347-353

16. Kalof AN, Cooper K (2007) Our approach to squamous intraepithelial lesions of the uterine cervix. J Clin Pathol 60:449-455

17. Kearsey SE, Labib K (1998) MCM proteins: evolution, properties, and role in DNA replication. Biochim Biophys Acta 1398:113-136

18. Klaes R, Benner A, Friedrich T et al (2002) p16INK4a immunohistochemistry improves interobserver agreement in the diagnosis of cervical intraepithelial neoplasia. Am J Surg Pathol 26:1389-1399

19. Klaes R, Friedrich T, Spitkovsky D et al (2001) Overexpression of p16(INK4 A) as a specific marker for dysplastic and neoplastic epithelial cells of the cervix uteri. Int J Cancer 92:276-284

20. Kruse AJ, Baak JP, Helliesen T et al (2002) Evaluation of MIB-1-positive cell clusters as a diagnostic marker for cervical intraepithelial neoplasia. Am J Surg Pathol 26:1501-1507

21. Kruse AJ, Skaland I, Janssen EA et al (2004) Quantitative molecular parameters to identify low-risk and high-risk early CIN lesions: role of markers of proliferative activity and differentiation and $\mathrm{Rb}$ availability. Int J Gynecol Pathol 23:100-109

22. Leong TY, Cooper K, Leong AS (2010) Immunohistology-past, present, and future. Adv Anat Pathol 17:404-418

23. Martin CM, O'Leary JJ (2011) Histology of cervical intraepithelial neoplasia and the role of biomarkers. Best Pract Res Clin Obstet Gynaecol 25:605615

24. McCluggage WG, Bharucha H, Caughley LM et al (1996) Interobserver variation in the reporting of cervical colposcopic biopsy specimens: comparison of grading systems. J Clin Pathol 49:833-835

25. Negri G, Bellisano G, Zannoni GF et al (2008) p16 ink4a and HPV L1 immunohistochemistry is helpful for estimating the behavior of low-grade dysplastic lesions of the cervix uteri. Am J Surg Pathol 32:1715-1720

26. Pirog EC, Baergen RN, Soslow RA et al (2002) Diagnostic accuracy of cervical low-grade squamous intraepithelial lesions is improved with MIB-1 immunostaining. Am J Surg Pathol 26:70-75

27. Quade BJ, Yang A, Wang Y et al (2001) Expression of the $\mathrm{p} 53$ homologue $\mathrm{p} 63$ in early cervical neoplasia. Gynecol Oncol 80:24-29

28. Sanati S, Huettner P, Ylagan LR (2010) Role of ProExC: a novel immunoperoxidase marker in the evaluation of dysplastic squamous and glandular lesions in cervical specimens. Int J Gynecol Pathol 29:79-87
29. Stoler MH, Schiffman M (2001) Interobserver reproducibility of cervical cytologic and histologic interpretations: realistic estimates from the ASCUS-LSIL Triage Study. JAMA 285:1500-1505

30. Tachibana KE, Gonzalez MA, Coleman N (2005) Cell-cycle-dependent regulation of DNA replication and its relevance to cancer pathology. J Pathol 205:123-129

31. Tsoumpou I, Arbyn M, Kyrgiou M et al (2009) p16(INK4a) immunostaining in cytological and histological specimens from the uterine cervix: a systematic review and meta-analysis. Cancer Treat Rev 35:210-220

32. Tzankov A, Went P, Zimpfer A, Dirnhofer S (2005) Tissue microarray technology: principles, pitfalls and perspectives-lessons learned from hematological malignancies. Exp Gerontol 40:737-744

33. Wells M, Östör AG, Crum CP et al (2003) Epithelial tumours. In: Tavassoli FA, Devilee P (Hsrg) Tumours of the Breast and Female Genital Organs. IARC Press, Lyon, S 259-290

34. Williams GH, Romanowski P, Morris L et al (1998) Improved cervical smear assessment using antibodies against proteins that regulate DNA replication. Proc Natl Acad Sci U S A 95:14932-14937

35. Hausen $\mathrm{H}$ zur (2002) Papillomaviruses and cancer: from basic studies to clinical application. Nat Rev Cancer 2:342-350 